\title{
Improved line frequencies for the nucleic acid base uracil for a radioastronomical search ${ }^{\star}$
}

\author{
S. Brünken ${ }^{1}$, M. C. McCarthy ${ }^{1,2}$, P. Thaddeus ${ }^{1,2}$, P. D. Godfrey ${ }^{3}$, and R. D. Brown ${ }^{3}$ \\ 1 Harvard-Smithsonian Center for Astrophysics, 60 Garden Street, Cambridge, MA 02138, USA \\ 2 Division of Engineering and Applied Sciences, Harvard University, 29 Oxford Street, Cambridge, MA 02138, USA \\ School of Chemistry, Monash University, Wellington Road, Clayton, Victoria 3800, Australia
}

Received 8 June 2006/ Accepted 26 July 2006

\section{ABSTRACT}

\begin{abstract}
Aims. We report new laboratory spectroscopic data on the rotational spectrum of the pyrimidine nucleic acid base uracil $\left(\mathrm{C}_{4} \mathrm{H}_{4} \mathrm{~N}_{2} \mathrm{O}_{2}\right)$. The dataset has been extended both into the microwave region and towards $\mathrm{mm}$-wavelengths with the aim of providing accurate transition rest frequencies for astrophysical searches.

Methods. The microwave measurements have been performed with a molecular beam Fourier transform microwave spectrometer in the frequency range from 9-19 GHz and the mm-wave band transitions have been recorded with a Stark-modulated free jet spectrometer up to $100 \mathrm{GHz}$. The global dataset has been analysed with a standard $S$-reduced Hamiltonian and precise spectroscopic parameters up to quartic order have been obtained. The hyperfine structure due to the two ${ }^{14} \mathrm{~N}$ nuclei has been resolved in the microwave measurements and the nuclear quadrupole coupling constants could be derived to high accuracy.

Results. Based on the new laboratory data and analysis, highly precise rotational transition rest frequencies are available for astrophysical important lines of uracil up to $300 \mathrm{GHz}$.
\end{abstract}

Key words. ISM: molecules - line: identification - molecular data - astrochemistry - ISM: lines and bands - radio lines: ISM

\section{Introduction}

More than 130 molecules have to date been identified in the interstellar medium (ISM), the great majority organic species. The formation of complex organic molecules and other so-called "prebiotic" molecules in extraterrestrial environments is a topic of lively debate (e.g. Ehrenfreund et al. 2002; Kwok 2004). Recently, the detection of the smallest amino acid glycine by means of mm-wavelength observations towards three massive star forming regions was claimed by Kuan et al. (2003a), but serious objections to this identification have been raised (Snyder et al. 2005; Ohishi 2006). Nevertheless, the detection of the simplest sugar, glycolaldehyde (Hollis et al. 2000), towards the galactic center source Sagittarius B2 and of a plethora of ketones and aldehydes (e.g. acetone, Snyder et al. 2002; propenal and propanal, Hollis et al. 2004b; alcohols, Turner \& Apponi 2001; Millar et al. 1988) and ethers (e.g. Turner 1991; Nummelin et al. 2000; Fuchs et al. 2005) towards different hot core regions provide evidence that large organic molecules are synthesized in the interstellar medium. The large variety of organics found in meteorites (e.g. Cooper et al. 2001) strengthens this notion.

The five nucleic acid bases, or nucleobases, the "alphabet" of the genetic code, are another important class of interesting prebiotic molecules. All five are small N-heterocyclic compounds, which can be divided into either purine (adenine, guanine) or pyrimidine (uracil, thymine, cytosine) derivatives. Uracil $\left(\mathrm{C}_{4} \mathrm{H}_{4} \mathrm{~N}_{2} \mathrm{O}_{2}\right.$, see Fig. 1), consisting of 12 atoms, is the smallest of the nucleobases and has been detected in both the

* Table 2 is only available in electronic form at the CDS via anonymous ftp to cdsarc.u-strasbg.fr $(130.79 .128 .5)$ or via http://cdsweb.u-strasbg.fr/cgi-bin/qcat? J/A+A/459/317
Murchison and Murray and the Orgueil meteorites (Stoks \& Schwartz 1979, 1981). The possible formation processes of nucleobases in the ISM have been discussed most recently by Peeters et al. (2003, 2005). These authors suggest that the most likely formation of uracil is by photoprocessing of pyrimidine (c- $\mathrm{C}_{4} \mathrm{H}_{4} \mathrm{~N}_{2}$ ) in water-ice containing dust grains, which links the expected interstellar uracil abundance to that of pyrimidine, which has not yet been detected in the ISM (Kuan et al. 2003b). The synthesis of uracil on icy dust grains may follow a similar pathway to that proposed in an aqueous environment on the prebiotic earth. This pathway involves reactions of cyanoacetylene $\left(\mathrm{HC}_{3} \mathrm{~N}\right)$ and $\mathrm{CN}$, which are both very abundant in interstellar sources (Ferris et al. 1968; Orgel 2004). Large organic molecules with aromatic structures are now widely believed to be the carriers of the UIR bands and to be fairly abundant in the interstellar medium. There is experimental evidence that nucleobases can form as by-products of PAH synthesis (Ricca et al. 2001; Bernstein et al. 2002). Once the compound is formed, it might be ejected into the interstellar gas by radiative or shock heating. Ejection may also occur in the hot cores of molecular clouds, when temperatures rise to $100-200 \mathrm{~K}$ and above.

In this paper, we present new laboratory data on the rotational spectrum of uracil with the aim of providing highly accurate transition frequencies for astrophysical searches to better than $0.3 \mathrm{~km} \mathrm{~s}^{-1}$ for frequencies up to $300 \mathrm{GHz}$. The existing dataset, consisting of 65 rotational transitions in the $58-63 \mathrm{GHz}$ range reported by Brown et al. (1988b), has been extended both to the astrophysically important frequency range between 70-100 GHz, where the strongest uracil lines are expected for interstellar sources with excitation temperatures around $10 \mathrm{~K}$, and towards the microwave region between $10-20 \mathrm{GHz}$. With the 

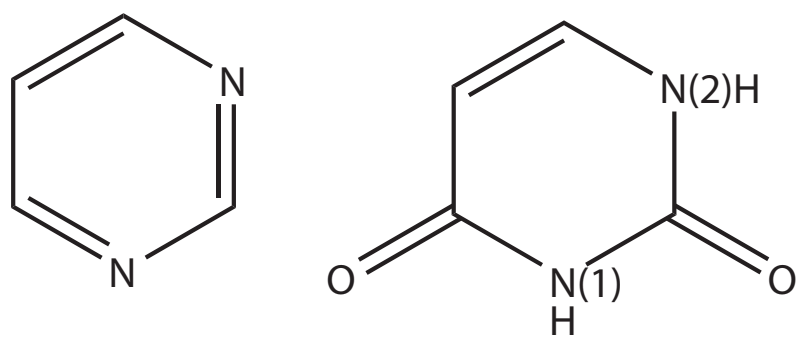

Fig. 1. Chemical structure of pyrimidine (left) and the lowest energy tautomer of uracil (right).

latter measurements it was possible to resolve and analyse the nuclear hyperfine structure due to the two ${ }^{14} \mathrm{~N}$ nuclei in uracil.

\section{Experimental setup}

The molecular beam Fourier transform microwave (FTM) spectrometer at Harvard was used to measure rotational transitions of uracil in the frequency range between 9 and $19 \mathrm{GHz}$. A detailed description of this instrument has been given previously (McCarthy et al. 1997). Uracil ( $\mathrm{mp} 338{ }^{\circ} \mathrm{C}$ ) is a solid with no significant vapour pressure at room temperature so two different techniques have been employed in this work to produce uracil in the gas phase. In the first, commercially available microcrystalline uracil powder (Alfa Aesar, 99+\%) was resistively heated in the presence of a stream of Ne gas. A pulsed seeded supersonic molecular beam with 300-400 $\mu$ s pulse duration was then produced by a commercial solenoid valve (General Valve) at a repetition rate of $6 \mathrm{~Hz}$ with a typical background pressure of $2.5 \mathrm{~atm}$ and an expansion parallel to the axis of the FabryPerot cavity (Thorwirth et al. 2005). Rotational temperatures are typically $1-2 \mathrm{~K}$ due to efficient rotational cooling in the supersonic expansion. Conditions were optimised on a strong hyperfine component of the $J_{K_{\mathrm{a}} K_{\mathrm{c}}}=2_{21}-1_{10}$ rotational transition of uracil. It was found that in the temperature range $150-180{ }^{\circ} \mathrm{C}$, the signal increased linearly with temperature, with a 6-fold increase over this temperature range ${ }^{1}$.

Laser ablation is a second technique to vapourize low volatile species. Recently this method has been combined with FTM spectrometers by several groups to obtain the microwave spectra of metal containing (Suenram et al. 1990; Hensel et al. 1993) and organic species (Kretschmer et al. 2003; Lesarri et al. 1996). In the same way, we recently incorporated a laser ablation source into our FTM spectrometer. The source consists of a Q-switched Nd:YAG laser (Minilite, Continuum) providing short (5 ns), intense (energy $28 \mathrm{~mJ}(12 \mathrm{~mJ})$ at $1064 \mathrm{~nm}(532 \mathrm{~nm})$ ) pulses which are focused on a rotating and translating rod (spot size $\sim 200-650 \mu \mathrm{m})$. The ablated material is seeded in an inert buffer gas (typically $\mathrm{Ne}$ ) and a supersonic molecular beam is produced by a solenoid valve (General Valve). Unlike the configuration adopted for the heated nozzle source, the molecular beam is orientated perpendicular to the axis of the FabryPerot cavity. Test measurements with OCS in Ne resulted in tenfold weaker lines in the perpendicular configuration than in the

1 The maximum operating temperature is limited to $\sim 180{ }^{\circ} \mathrm{C}$; a higher temperature will cause permanent damage to the solenoid assembly.

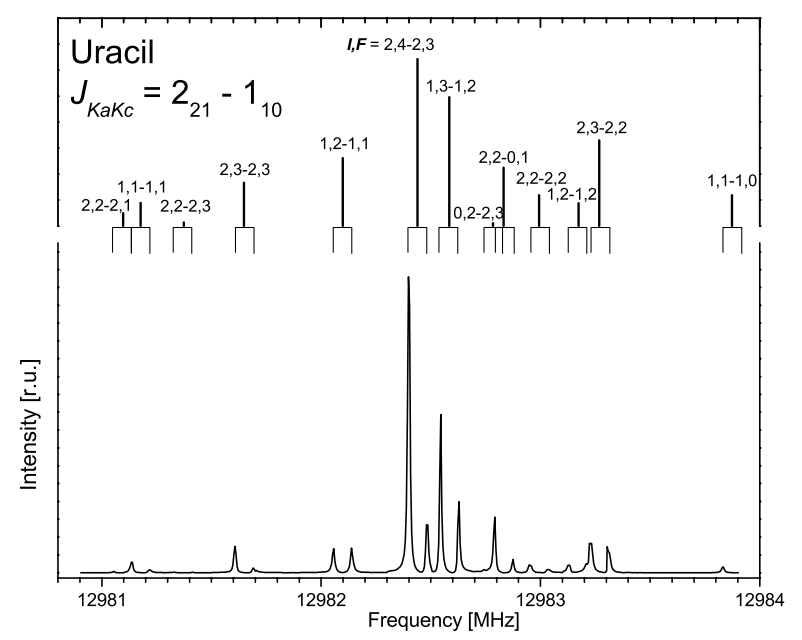

Fig. 2. Top: calculated stick diagram of the $J_{K_{\mathrm{a}} K_{\mathrm{c}}}=22_{21}-1_{10}$ rotational transition. Bottom: experimental spectrum recorded with the FTMW spectrometer. Each hyperfine transition consists of a Doppler splitted doublet due to the supersonic velocity of the molecular beam relative to the two traveling waves that compose the confocal mode of the Fabry Perot cavity.

parallel $^{2}$. The uracil samples were prepared by pressing the microcrystalline powder with small amounts $(<1 \%)$ of a commercial binder into a rod $6 \mathrm{~mm}$ in diameter and $24 \mathrm{~mm}$ long. A significant increase in signal strength, by a factor of $4-5$, is found for the latter source, in spite of the unfavourable geometrical arrangement.

The Stark-modulated free-jet spectrometer used for the mmwave studies has been previously described (Brown et al. 1988a) but was modified to replace klystrons with solid-state oscillators, coverage being extended to an upper frequency limit of $100 \mathrm{GHz}$. Uracil has been seeded in an argon continuous flow supersonic nozzle beam after its evaporation from a microcrystalline powder at temperatures of around $300-350^{\circ}$. The supersonic expansion yields rotational temperatures of about $10 \mathrm{~K}$.

\section{Measurements, analysis and results}

In total, 34 new rotational transitions have been measured in the microwave region between $9-19 \mathrm{GHz}$ and 43 in the mm-wave band between $73-100 \mathrm{GHz}$. The microwave transitions consist of $6 a$ - and $28 b$-type transitions covering the energy range $E_{\text {lower }}=0.17-4.74 \mathrm{~cm}^{-1}$, with quantum numbers up to $J=8$ and $K_{\mathrm{a}}=4$. Line frequency uncertainties range from $2-5 \mathrm{kHz}$, the higher value resulting from the increased experimental linewidth in the perpendicular mode of the FTM spectrometer. The hyperfine structure from the two ${ }^{14} \mathrm{~N}$ nuclear quadrupole moments has been partly resolved for these transitions. The assignment has been facilitated by ab initio calculations of the nuclear quadrupole coupling tensor after optimization of the molecular structure at the B3LYP/cc-pVTZ level of theory with Gaussian98 (Frisch et al. 1998). As an example the calculated stick diagram and measured spectrum of the $J_{K_{3} K_{\mathrm{c}}}=2_{21}-$ $1_{10}$ rotational transition is shown in Fig. 2 . The newly recorded

\footnotetext{
2 The perpendicular arrangement was chosen because of space limitations behind the cavity mirror. A refined system with the laser ablation source on the axis of the cavity is under construction.
} 
Table 1. Rotational and hyperfine interaction constants for uracil. Values in parentheses represent one standard deviation in units of last digit.

\begin{tabular}{|c|c|c|c|c|}
\hline Parameter & & $\begin{array}{c}\text { This work } \\
\text { experimental }\end{array}$ & Brown et al. (1988b) & $\begin{array}{l}\text { This work } \\
\text { ab initio }{ }^{a}\end{array}$ \\
\hline$A$ & $\mathrm{MHz}$ & $3883.873021(60)$ & $3883.87825(110)$ & 3902.4 \\
\hline$B$ & $\mathrm{MHz}$ & $2023.732581(45)$ & $2023.73267(101)$ & 2022.2 \\
\hline$C$ & $\mathrm{MHz}$ & $1330.928108(33)$ & $1330.92380(60)$ & 1332.0 \\
\hline$D_{J}$ & $\mathrm{kHz}$ & $0.06336(44)$ & $0.06029(77)$ & \\
\hline$D_{J K}$ & $\mathrm{kHz}$ & $0.10551(232)$ & $0.1047(14)$ & \\
\hline$D_{K}$ & $\mathrm{kHz}$ & $0.4530(32)$ & $0.4724(43)$ & \\
\hline$d_{1}$ & $\mathrm{kHz}$ & $-0.026229(179)$ & $-0.02738(28)$ & \\
\hline$d_{2}$ & $\mathrm{kHz}$ & $-0.006803(132)$ & $-0.006532(94)$ & \\
\hline$\chi_{a a}(\mathrm{~N} 1)$ & $\mathrm{MHz}$ & $1.92548(241)$ & & 2.049 \\
\hline$\chi_{b b}(\mathrm{~N} 1)$ & $\mathrm{MHz}$ & $1.52731(318)$ & & 1.684 \\
\hline$\chi_{a a}(\mathrm{~N} 2)$ & $\mathrm{MHz}$ & $1.76000(247)$ & & 1.880 \\
\hline$\chi_{b b}(\mathrm{~N} 2)$ & $\mathrm{MHz}$ & $1.98111(288)$ & & 2.069 \\
\hline$\mu_{a}$ & $\bar{D}$ & & 1.61 & 1.40 \\
\hline$\mu_{b}$ & D & & 3.52 & 4.29 \\
\hline $\begin{array}{l}\text { No. lines } \\
\text { wrms }\end{array}$ & & $\begin{array}{r}142 \\
1.1\end{array}$ & & \\
\hline
\end{tabular}

${ }^{a}$ The structure was optimized at the B3LYP/cc-pVTZ level of theory, the nuclear quadrupole coupling constants were calculated using the epr-iii basis set.

mm-wavelength $b$-type transitions include rotational energy levels up to $J=18$ and $K_{\mathrm{a}}=12$ and $E_{\text {lower }}=22.82 \mathrm{~cm}^{-1}$, and have an experimental unertainty of typically $50 \mathrm{kHz}$. A complete linelist of all new and previous rotational data on uracil can be found in the electronic supplement to this article (Table 2).

Uracil is a planar asymmetric rotor molecule $(\kappa=-0.46)$ with comparatively large electric dipole moment components of $\mu_{a}=1.61 \mathrm{D}$ and $\mu_{b}=3.52 \mathrm{D}$ along the $a$ and $b$ principal axes, respectively (Brown et al. 1988b). The new data together with all previously reported $\mathrm{mm}$-wavelength lines have been analysed with a standard $S$-reduced Watson type Hamiltonian in the $I^{r}$ representation with Pickett's spfit/spcat program suite (Pickett 1991). Nuclear quadrupole interaction terms $\chi_{i i}(i=a, b, c)$ have been included for both ${ }^{14} \mathrm{~N}$ nuclei, and since they are of the same order of magnitude, a coupling scheme was used where the two nuclear spins $\boldsymbol{I}_{\mathrm{N} 1}$ and $\boldsymbol{I}_{\mathrm{N} 2}\left(\boldsymbol{I}_{\mathrm{N} 1}=\boldsymbol{I}_{\mathrm{N} 2}=1\right)$ are first coupled to form the resultant spin $\boldsymbol{I}=\boldsymbol{I}_{\mathrm{N} 1}+\boldsymbol{I}_{\mathrm{N} 2}$ which then couples to the molecular rotation to yield the total angular momentum $\boldsymbol{F}=\boldsymbol{J}+\boldsymbol{I}$.

The derived spectroscopic parameters are summarized in Table 1 together with values previously reported (Brown et al. $1988 b)$ and with results from our ab initio calculations. The molecular constants $A, B$ and $C$ derived in this work are better by a factor of $\sim 20$. The improvement in the centrifugal distortion parameters is less marked, since the new dataset does not add significantly higher $J$ and $K_{\mathrm{a}}$ levels to the analysis. The nuclear quadrupole coupling constants are derived for the first time and determined to better than $1 \%$. The ab initio calculations overestimated these values by around $10 \%$. The wrms (weighted root mean square) value of the fit of 1.1 indicates that the observed transition frequencies are generally reproduced to within their experimental uncertainties.

\section{Discussion}

The improved spectroscopic parameter set obtained in the present analysis allows for precise predictions of transition rest frequencies of uracil to better than $0.3 \mathrm{~km} \mathrm{~s}^{-1}$ covering the astrophysically important frequency range up to $300 \mathrm{GHz}$. Predicted rotational spectra for excitation temperatures of $10 \mathrm{~K}$ and $150 \mathrm{~K}$ are shown in Fig. 3. At a temperature of $10 \mathrm{~K}$ the line intensity

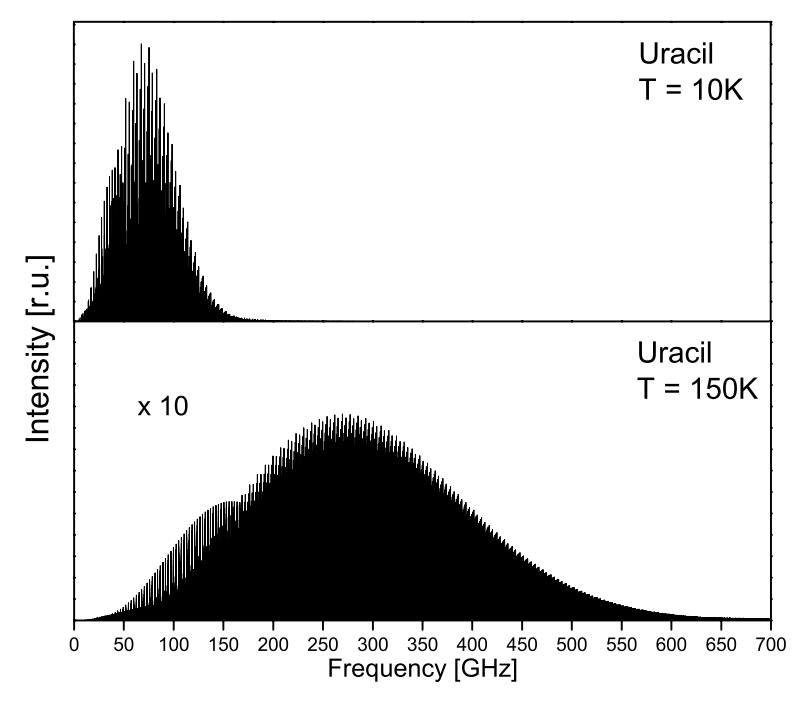

Fig. 3. Calculated stick diagram of the rotational spectrum of uracil, neglecting hyperfine structure at a temperature of $10 \mathrm{~K}$ (top) and $150 \mathrm{~K}$ (bottom).

peaks at around $60-80 \mathrm{GHz}$. The strongest lines are $b$-type $\left(\Delta K_{\mathrm{a}}=\Delta K_{\mathrm{c}}=1\right) R$-branch $(\Delta J=+1)$ transitions involving quantum numbers $J=7-13$ and $K_{\mathrm{a}}=7-13$ with $K_{\mathrm{a}}=$ $J, J-1, \ldots, J-4$. The calculated frequency uncertainty of these lines is less than $10 \mathrm{kHz}$ or $0.05 \mathrm{~km} \mathrm{~s}^{-1}$. At a typical hot core temperature of $150 \mathrm{~K}$ the intensity maximum shifts towards a frequency of $270 \mathrm{GHz}$. It should be noted that intensities are generally decreased by a factor of more than 10 owing to the larger rotational partition function. The strongest lines in this case are $b$-type $R$-branch transitions with $J=31-40, K_{\mathrm{a}}=31-40$ and $K_{\mathrm{a}}=J, J-1, J-2$, with calculated frequency uncertainties of less than $300 \mathrm{kHz}$ or $0.3 \mathrm{~km} \mathrm{~s}^{-1}$. Although these values might be underestimated owing to missing higher order centrifugal terms in 
the Hamiltonian, even frequency uncertainties a factor 5 higher would be sufficient for a secure line identification in sources with broad line emission of the order of $5-10 \mathrm{~km} \mathrm{~s}^{-1}$.

The best interstellar sources in which to search for uracil are probably those rich in other complex organic species containing $\mathrm{C}, \mathrm{N}$ and $\mathrm{O}$, specifically the hot cores of massive star forming regions like Orion KL, W51e1/e2 or Sgr B2(N-LMH) and the hot cores ("corinos") of low mass star forming regions like IRAS16293-2422 (Cazaux et al. 2003). However, rotational excitation temperatures in these sources are typically of the order of 100-200 K, which in combination with the high partition function of uracil at these temperatures results in rather low line intensities. Recently, Hollis et al. found evidence for an extended $\left(>60^{\prime \prime}\right)$, cold $\left(T_{\text {rot }}=8 \mathrm{~K}\right)$ halo around Sgr B2(N) through observations with the $100 \mathrm{~m}$ Green Bank Telescope (GBT) of cm-wavelength emission and absorption lines of glycolaldehyde and acetamide (Hollis et al. 2004a, 2006). In such a cold environment rotational transitions of uracil peak in the $3 \mathrm{~mm}$ band and its gas phase emission spectrum might be observable, provided that an efficient formation and ejection process exists, possibly one caused by shocks. Based on these assumptions, we searched for uracil emission lines in two recent line surveys of the Sgr B2(N) region by Apponi (2006) and Belloche (2006) performed with the Kitt Peak 12 m and IRAM 30 m radio telescopes, respectively, and obtained an upper limit for the uracil column density of $N=6.0 \times 10^{13} \mathrm{~cm}^{-2}$, which is only slightly less than the pyrimidine upper limit of $1.7 \times 10^{14} \mathrm{~cm}^{-2}$ in this source (Kuan et al. 2003b). Out of the 18 lines which fall within the range of the surveys, 13 coincide with emission lines and 2 with absorption features. However, since at 3 additional line positions no significant features could be detected above the noise level, these coincidences appear to be accidental ${ }^{3}$. This finding is another example of the problem of line confusion in line-rich sources as Sgr B2(N) at mm-wavelengths, which has been recently discussed in the context of the detection of other large organic species (Snyder et al. 2005; Apponi et al. 2006). Spectral line confusion is less pronounced in the $\mathrm{cm}$-wavelength region, and the capability of detecting complex molecules in Sgr B2(N) at cm-wavelengths has been recently demonstrated by GBT observations of acetamide, propenal and propanal (Hollis et al. 2004b, 2006). Astrophysical searches for uracil transitions in the 12-15 GHz region with the GBT towards Sgr B2(N) are scheduled. Additional advantages in this frequency region are the possible observation of rotational lines in absorption against strong continuum background sources and a secured identification owing to resolved hyperfine splitting in low $J$ rotational lines.

This work provides highly accurate transition frequencies of uracil up to $300 \mathrm{GHz}$, which allows for deep astronomical searches for this molecule. A detailed tabulation of uracil transition frequencies derived from the present analysis will be available in the Cologne Database for Molecular Spectroscopy (Müller et al. 2005) at http: //www . cdms . de.
Acknowledgements. The authors thank J.-U. Grabow, M. E. Sanz and J. L. Alonso for helpful discussions regarding the preparation of sample rods; A. Apponi and A. Belloche for providing their survey data prior to publication; C. Gottlieb, H. Gupta, Z. Yu, H. S. P. Müller and T. Giesen for helpful discussions; and E. S. Palmer for laboratory assistance. S. B. is grateful to the Harvard College Observatory for the 2005 Menzel fellowship.

\section{References}

Apponi, A. 2006, private communication

Apponi, A. J., Halfen, D. T., Ziurys, L. M., et al. 2006, ApJ, 643, L29

Belloche, A. 2006, private communication

Bernstein, M. P., Elsila, J. E., Dworkin, J. P., et al. 2002, ApJ, 576, 1115

Brown, R. D., Crofts, J. G., Godfrey, P. D., McNaughton, D., \& Pierlot, A. P. 1988a, J. Mol. Struct., 190, 185

Brown, R. D., Godfrey, P., McNaughton, D., \& Pierlot, A. P. 1988b, J. Am. Chem. Soc, 110, 2329

Cazaux, S., Tielens, A. G. G. M., Ceccarelli, C., et al. 2003, ApJ, 593, L51

Cooper, G., Kimmich, N., Belisle, W., et al. 2001, Nature, 414, 879

Ehrenfreund, P., Irvine, W., Becker, L., et al. 2002, Rep. Prog. Phys., 65, 1427

Ferris, J. P., Sanchez, R. A., \& Orgel, L. 1968, J. Molec. Bio., 33, 693

Frisch, M. J., Trucks, G. W., Schlegel, H. B., et al. 1998, Gaussian 98, Gaussian Inc., Pittsburgh PA

Fuchs, G. W., Fuchs, U., Giesen, T. F., \& Wyrowski, F. 2005, A\&A, 444, 521

Hensel, K. D., Styger, C., Jäger, W., Merer, A. J., \& Gerry, M. C. L. 1993, J. Chem. Phys., 99, 3320

Hollis, J. M., Lovas, F. J., \& Jewell, P. R. 2000, ApJ, 540, L107

Hollis, J. M., Jewell, P. R., Lovas, F. J., \& Remijan, A. 2004a, ApJ, 613, L45

Hollis, J. M., Jewell, P. R., Lovas, F. J., Remijan, A., \& Møllendal, H. 2004b, ApJ, 610, L21

Hollis, J. M., Lovas, F. J., Remijan, A. J., et al. 2006, ApJ, 643, L25

Kretschmer, U., Consalvo, D., Knaack, A., et al. 2003, Rev. Sci. Instrum., 74, 4799

Kuan, Y., Charnley, S. B., Huang, H., Tseng, W., \& Kisiel, Z. 2003a, ApJ, 593, 848

Kuan, Y., Yan, C., Charnley, S. B., et al. 2003b, Mon. Not. R. Astron. Soc., 345, 650

Kwok, S. 2004, Nature, 430, 985

Lesarri, A., Mata, S., Lopez, J. C., \& Alonso, J. L. 1996, Mol. Phys., 87, 1159 McCarthy, M. C., Travers, M. J., Kovacs, A., Gottlieb, C. A., \& Thaddeus, P. 1997, ApJS, 113, 105

Millar, T. J., Brown, P. D., Olofsson, H., \& Hjalmarson, A. 1988, A\&A, 205, L5 Müller, H. S. P., Schlöder, F., Stutzki, J., \& Winnewisser, G. 2005, J. Mol. Struct., 742,215

Nummelin, A., Bergman, P., Hjalmarson, Å., et al. 2000, ApJS, 128, 213

Ohishi, M. 2006, private communication

Orgel, L. E. 2004, Crit. Rev. Biochem. Mol., 39, 99

Peeters, Z., Botta, O., Charnley, S. B., Ruiterkamp, R., \& Ehrenfreund, P. 2003, ApJ, 593, L129

Peeters, Z., Botta, O., Charnley, S. B., et al. 2005, A\&A, 433, 583

Pickett, H. M. 1991, J. Mol. Spectrosc., 148, 371

Ricca, A., Bauschlicher, C. W., \& Bakes, E. L. O. 2001, Icarus, 154, 516

Snyder, L. E., Lovas, F. J., Mehringer, D. M., et al. 2002, ApJ, 578, 245

Snyder, L. E., Lovas, F. J., Hollis, J. M., et al. 2005, ApJ, 619, 914

Stoks, P. G., \& Schwartz, A. W. 1979, Nature, 282, 709

Stoks, P. G., \& Schwartz, A. W. 1981, Geochim. Cosmochim. Ac., 45, 563

Suenram, R. D., Lovas, F. J., Fraser, G. T., \& Matsumura, K. 1990, J. Chem. Phys., 92, 4724

Thorwirth, S., McCarthy, M. C., Gottlieb, C. A., et al. 2005, J. Chem. Phys., 123, 4326

Turner, B. E. 1991, ApJS, 76, 617

Turner, B. E., \& Apponi, A. J. 2001, ApJ, 561, L207

\footnotetext{
3 It is from those undetected lines that the column density limit has been obtained, assuming $T_{\text {rot }}=10 \mathrm{~K}, \Delta v=20 \mathrm{~km} \mathrm{~s}^{-1}$ and an extended source relative to the beamsize of the telescopes.
} 